\title{
Problemas Internalizantes e Externalizantes de Crianças e Adolescentes com Fibrose Cística
}

\author{
Carla Cristina Borges ${ }^{1}$ \\ ${ }^{1}$ Universidade Federal de São Paulo, SP, Brasil.
}

\author{
Nancy Ramacciotti de Oliveira-Monteiro ${ }^{1}$
}

${ }^{1}$ Universidade Federal de São Paulo, SP, Brasil.

Resumo: A fibrose cística é uma doença genética, ainda sem cura, provocada por mutações cromossômicas, que pode afetar vários sistemas, dentre os quais o respiratório e o digestivo são os mais comumente atingidos. O adoecimento crônico traz alterações psicológicas para os pacientes e seus cuidadores. Com o objetivo de avaliar problemas internalizantes e externalizantes, e também competências de crianças e adolescentes com fibrose cística, foram entrevistados 31 cuidadores familiares, majoritariamente mães de pacientes na faixa etária de 6 a 18 anos, em salas de espera de três centros de referência no tratamento da doença na cidade de São Paulo. Os instrumentos utilizados foram: Inventário de Comportamentos da Infância e da Adolescência e diário de campo. Os resultados apontaram a prevalência de problemas internalizantes em adolescentes com fibrose cística. A análise do diário de campo indicou dificuldades na adesão ao tratamento e demandas de atendimento psicológico não assistidas em pacientes e seus cuidadores familiares. A ausência de profissional de Psicologia nas equipes multiprofissionais configurou-se como um prejuízo frente as condições psicológicas dos pacientes de fibrose cística e seus cuidadores familiares.

Palavras-chave:Fibrose cística, Problemasinternalizantes, Problemas externalizantes, Psicologia do desenvolvimento, Child behavior checklist.

\section{Internalizing and Externalizing Problems of Children and Adolescents with Cystic Fibrosis}

\begin{abstract}
Cystic fibrosis is a genetic disease, still without cure, caused by chromosomal mutations that can affect various systems, the respiratory and digestive systems being the most common. Chronic illness brings psychological changes to patients and their caregivers. Aiming to evaluate internalizing and externalizing problems, and competences of children and adolescents with cystic fibrosis, we interviewed 31 family caregivers, mostly mothers, of patients aged 6 to 18 years in waiting rooms of three Reference Centers in the treatment of the disease in the municipality of São Paulo, state of São Paulo. The instruments used were: Child behavior checklist and Field Diary. The results pointed out the prevalence of internalizing problems in patients with cystic fibrosis in adolescence. Field diary analysis indicated difficulties in adherence to treatment and unassisted demands for psychological care in patients and their family caregivers. The absence of a Psychology professional in the multiprofessional teams showed to be prejudicial to the psychological conditions of cystic fibrosis patients and their family caregivers.
\end{abstract}

Keywords: Cystic fibrosis, Internalizing problems, Externalizing problems, Developmental psychology, Child behavior checklist. 


\title{
Problemas de Internalización y Externalización de Niños y Adolescentes con Fibrosis Quística
}

\begin{abstract}
Resumen: La fibrosis quística es una enfermedad genética, aún sin cura, causada por mutaciones cromosómicas y que puede afectar varios sistemas, entre ellos los sistemas respiratorio y digestivo son los más comunes. La enfermedad crónica trae cambios psicológicos a los pacientes y sus cuidadores. Para evaluar los problemas de internalización y externalización, así como las competencias de niños y adolescentes con fibrosis quística, se entrevistó a 31 cuidadores familiares, en su mayoría madres de pacientes de 6 a 18 años de edad, en salas de espera de tres centros de referencia en el tratamiento de la enfermedad en la ciudad de São Paulo. Los instrumentos utilizados fueron: Inventario del Comportamiento de Niños y Adolescentes y diario de campo. Los resultados mostraron la prevalencia de problemas de internalización en pacientes con fibrosis quística en la adolescencia. El análisis del diario de campo indicó dificultades en la adherencia al tratamiento y demandas de asistencia psicológica no asistida en pacientes y sus cuidadores familiares. Se hace necesario un profesional de psicología en los equipos multiprofesionales ante las condiciones psicológicas de los pacientes con fibrosis quística y sus cuidadores familiares.
\end{abstract}

Palabras clave: Fibrosis quística, Problemas de internalización, Problemas de externalización, Psicología del desarrollo, Child behavior checklist.

\section{Introdução}

Após o reconhecimento da fibrose cística (FC) pela comunidade científica, em 1938, com os estudos da estadunidense Dorothy H. Andersen, da Universidade de Colúmbia, outro marco na história da doença ocorreu em 1946. Nesse ano, estudos genéticos com pacientes e familiares concluíram que a doença era de herança recessiva e que sua causa encontrava-se em um único gene. Desde a década de 1980, sabe-se que a FC é uma doença crônica de origem genética, de comprometimento multissistêmico, progressivo e fatal. A doença tem herança autossômica recessiva, o que, em termos práticos, significa que sua ocorrência depende de um componente genético presente em ambos os genitores. Quando pai e mãe são portadores, a probabilidade de o filho nascer com a doença é de 25\% (Cabello, 2011).

A FC desenvolve-se a partir de uma mutação no cromossomo 7, que acarreta uma produção deficitária da proteína CFTR (Cystic Fibrosis Transmembrane Conductance Regulator), que leva o mesmo nome do gene responsável pela doença. Dentre os sistemas do corpo que podem ser afetados pela FC, o respiratório e o digestivo são os mais frequentes. Por ter um caráter de comprometimento multissistêmico, a FC exige um tratamento complexo que envolve a ação de diferentes profissionais da saúde (Grupo Brasileiro de Estudos de Fibrose Cística [GBEFC], 2016).

No Brasil, o Grupo Brasileiro de Estudos de FC (GBEFC), que existe desde 2003, realiza o levantamento da quantidade e das condições clínicas de pacientes atendidos nos Centros de Referência no Tratamento da FC (CRTs) (GBEFC, 2015). Um CRT atende no mínimo 50 pacientes regularmente e apresenta estrutura para diagnóstico, seguimento e tratamento de pacientes de FC com equipe multiprofissional (Athanazio et al., 2017).

Desde 2009, o GBEFC compartilha on-line relatórios anuais elaborados a partir de informações fornecidas por CRTs de todo o Brasil, apresentando dados epidemiológicos e sobre condições clínicas de pacientes com FC. Até o final de 2017, os CRTs brasileiros tinham 5.128 pacientes registrados, dos quais 474 foram diagnosticados naquele ano. Do total de pacientes com registro de FC em todo o país, $71,1 \%$ eram menores de 18 anos (GBEFC, 2017).

A prevalência da faixa etária pediátrica entre os pacientes de FC é apontada como realidade de algumas regiões no mundo, entre elas, a América Latina. Comparativamente, nos Estados Unidos, em 2017, 
dos 30.139 pacientes, $46,4 \%$ deles eram menores de 18 anos (Cystic Fibrosis Foundation [CFF], 2018). Para Lentini et al. (2014), os piores resultados na América Latina decorrem do menor nível socioeconômico dessas populações, do acesso desigual à saúde, do baixo orçamento para saúde e da carência de organizações especializadas no tratamento da doença.

Em relação aos pacientes atendidos no Brasil, conforme todos os relatórios do GBEFC desde 2009, a maior concentração deles encontra-se na região Sudeste $(47,5 \%)$, sendo que o estado de São Paulo apresenta a maior quantidade em todo o país, com $27,4 \%$ (GBEFC, 2017). A maior concentração de CRTs nessa região pode estar associada à prevalência de pacientes. Além disso, condições de tratamento e, sobretudo, de diagnóstico são essenciais à ocorrência de novos registros de pacientes e oferecimento de assistência adequada. Embora em todas as regiões brasileiras haja CRTs, a distribuição deles pelo país ainda é desigual (Borges, Bezerra, \& Scaff, 2019). Na região Norte, por exemplo, existem CRTs apenas nas capitais dos estados do Pará e Amazonas, quantidade inferior aos seis CRTs existentes somente da capital paulista (GBEFC, 2017).

A inclusão da testagem para a FC na triagem neonatal, popularmente conhecida como teste do pezinho, contribui com a realização de novos diagnósticos da doença, de forma precoce. Em 2017, os diagnósticos de FC via teste do pezinho tiveram média de idade de 0,44 anos, enquanto entre os realizados a partir de sintomas clínicos, a média foi de 8,65 anos (GBEFC, 2017). Um diagnóstico dito precoce, ou seja, aquele que acontece ainda nos primeiros meses de vida, pode implicar no início da assistência ao paciente de FC, o que é fundamental para sua sobrevivência, além de promover melhores condições clínicas em comparação aos pacientes diagnosticados com idade mais avançada (D’Alessandro, Rentería, Fernández, Martínez, \& Segal, 2009). No entanto, apesar da importância da testagem para FC na triagem neonatal, em 2017, os diagnósticos via sintomas clínicos persistentes, sobretudo os respiratórios, ainda foram mais abundantes $(56,9 \%)$ em relação aos detectados via teste do pezinho $(35,4 \%)$. Dentre o total de pacientes com FC registrados no Brasil, a média de idade diagnóstica é de 5,74 anos (GBEFC, 2017).

$\mathrm{O}$ adoecimento crônico em FC insere a família em um contexto inesperado de rotinas de internações, medicamentos, dieta diferenciada e sessões de fisioterapia, além das inúmeras possíveis limitações quanto à realização de atividades físicas com as quais o paciente e sua família precisam conviver (Afonso \& Mitre, 2013). Desgastes físicos e psicológicos podem acometê-los, em decorrência de alterações no cotidiano familiar causadas especialmente pelas adaptações às necessidades do tratamento e também pela adequação às regras das instituições de saúde (Afonso, Gomes, \& Mitre, 2015).

É comum falar-se em balanço energético negativo da FC, já que o organismo acometido por essa doença consome mais energia do que absorve, o que faz com que o paciente necessite de uma dieta hipercalórica (Chaves, Cunha, Costa, Costa, \& Lacerda, 2015, p. 3325). Além de condições nutricionais adequadas, a estabilidade das condições clínicas do paciente de FC depende do comparecimento às consultas médicas e de outras profissões da saúde, sessões de fisioterapia respiratória, uso adequado de medicamentos e períodos de internação, conforme necessidade do paciente (Adde, 2015; Conto et al., 2014; Griese \& Kappler, 2008). No entanto, mesmo dispondo de orientações adequadas, são necessários recursos, materiais ou psicológicos, para assistir o paciente, proporcionando, assim, o melhor aproveitamento possível do tratamento, com consequente melhora em sua qualidade de vida (Castro \& Piccinini, 2002).

Dentre os problemas de adesão ao tratamento da FC, destacam-se aspectos de ordem psicológica nos pacientes (Mello \& Moreira, 2010). Fala-se em uma espécie de esgotamento psíquico, seja da família seja do paciente que, a todo momento, precisa lidar com a necessidade de um tratamento complexo. A negligência ao tratamento pode ter como consequências a piora do estado clínico ou até mesmo a morte (Tavares, Carvalho, \& Pelloso, 2010; Tavares, Carvalho, \& Pelloso, 2014). O último relatório disponível para consulta, até o momento da redação deste trabalho, o referente ao ano de 2017, relatou 50 óbitos de pacientes com $\mathrm{FC}$, dos quais $86 \%$ foram decorrentes de causas respiratórias e cuja média de idade era de 17,4 anos. Esse relatório ainda apresentou uma idade de estimativa de sobrevida da população brasileira de pacientes de FC de 41,6 anos (GBEFC, 2017).

Em 2015, o trabalho de Quittner et al. (2016), desenvolvido a partir da parceria entre a CFF e a European Cystic Fibrosis Society (ECFS), salientou a importância de avaliações de saúde mental em pacientes de FC e seus cuidadores. Foi criado um 
comitê internacional de saúde mental na FC, composto por 22 membros de diferentes formações e experiências no universo de adoecimento e tratamento da doença, entre eles: médicos, psicólogos, pacientes e cuidadores familiares de pacientes de FC. Esse estudo ressaltou, ainda, que ansiedade e depressão são as principais demandas psicológicas verificadas nessa população, cujos índices são maiores à medida que o paciente envelhece. Comparativamente à população sem a doença, os pacientes de FC, a partir da adolescência, apresentaram risco de duas a três vezes maior de desenvolver ansiedade e depressão. $\mathrm{E}$ em análises de díades formadas com pacientes com FC e seus pais, diante de ansiedade e depressão parental, a probabilidade de o filho também apresentar uma dessas condições psicológicas pertinentes a problemas internalizantes foi pelo menos duas vezes maior em comparação àqueles cujos pais não apresentaram ansiedade ou depressão (Quittner et al., 2016).

Ainda sobre o estudo de Quittner et al. (2016), o Comitê Internacional em Saúde Mental na FC elaborou 15 diretrizes de avaliação e tratamento voltadas a pacientes com FC e seus cuidadores familiares, no sentido de orientar os centros de atendimento da doença quanto ao manejo da ansiedade e depressão. Segundo essas diretrizes, o acompanhamento deve ser contínuo, com avaliações anuais de saúde mental tanto em pacientes quanto em pelo menos um cuidador familiar. Na faixa etária de 7 a 11 anos, a avaliação deve ocorrer com os cuidadores, mas a partir dos 12 anos, essa avaliação deve incluir o paciente, com instrumentos próprios à faixa etária. Quadros de ansiedade e depressão nos pacientes devem ser manejados pela equipe do centro de atendimento de FC, prioritariamente através de intervenção psicológica. $\mathrm{O}$ uso de medicamentos deve ser considerado somente em casos em que a intervenção psicológica mostrar-se ineficaz e deve ser acompanhado por profissionais com larga experiência no tratamento da FC, dada a complexidade de uso de medicamentos que o paciente já utiliza (Quittner et al., 2016).

Neste estudo, o termo "condições psicológicas" refere-se a aspectos individuais relacionados às formas como o indivíduo lida com as exigências de sua vida, aspectos que são associados a competências (recursos) e a problemas psicológicos, que podem ser internalizantes (de ordem emocional) e/ ou externalizantes (comportamentais) (Bordin et al., 2013).
As competências referem-se ao funcionamento adaptativo do indivíduo frente às necessidades de seu cotidiano. Os problemas internalizantes dizem respeito a dificuldades emocionais, aquelas do "mundo interno" do indivíduo, como ansiedade e depressão. Já os problemas externalizantes são aqueles de natureza comportamental, com dificuldades associadas ao "mundo externo", como nos casos de agressividade e dificuldades de autocontrole (Achenbach, Ivanova, Rescorla, Turner, \& Althoff, 2016). Essa terminologia (internalizantes / externalizantes), enquanto tipos de problemas psicológicos, é adotada em estudos que utilizam o Sistema de Avaliação Empiricamente Baseado de Achenbach (ASEBA) (Achenbach \& Rescorla, 2010). Os instrumentos do ASEBA compõem um sistema padronizado e mundialmente utilizado de avaliação de funcionamento adaptativo/competência social e problemas comportamentais/emocionais em diferentes faixas etárias (Achenbach \& Rescorla, 2001). Essa avaliação multicultural, baseada em evidências, também permite comunicação e colaboração entre diferentes profissionais de saúde mental e pesquisadores ao redor do mundo, além de ser um instrumental qualificado para serviços que adotam práticas psicológicas baseadas em evidências (Achenbach, 2015).

Face ao exposto, o objetivo deste estudo foi avaliar competências e problemas internalizantes e externalizantes de crianças e adolescentes com FC, a partir do relato de seu cuidador familiar, verificando tais condições em termos de faixa etária (criança e adolescente) e sexo (feminino e masculino).

\section{Método}

\section{Participantes}

Participaram da pesquisa 31 cuidadores familiares de crianças e adolescentes, de 6 a 18 anos, com diagnóstico de FC, atendidos em três CRTs da cidade de São Paulo (SP). Dentre eles, 26 eram mães, 2 eram pais, 2 eram avós e 1 era padrasto. Os participantes foram convidados a participar do estudo entre novembro de 2016 e junho de 2017, nas salas de espera dos CRTs, enquanto aguardavam pela consulta médica do paciente pelo qual era responsável.

\section{Instrumentos}

Utilizou-se o Inventário de Comportamento da Infância e da Adolescência (Child behavior checklist 
(for ages 6-18) [CBCL]) do Sistema de Avaliação Empiricamente Baseado de Achenbach (Achenbach System of Empirically Based Assessment [ASEBA]), de Achenbach e Rescorla (2010). Trata-se de um instrumento organizado em duas seções, que avaliam: a) Competências sociais/funcionamento adaptativo; e b) Perfil de problemas psicológicos (internalizantes e externalizantes). A primeira seção engloba 20 itens, que recebem pontuação de zero a quatro, e avalia três aspectos: Atividades, Sociabilidade e Área escolar. $\mathrm{Na}$ segunda seção, investiga-se o perfil comportamental/problemas psicológicos a partir de 118 itens que recebem pontuações de zero a dois, sendo zero atribuído a "não é verdadeiro", um a "um pouco ou algumas vezes verdadeiro" e dois a "muito ou frequentemente verdadeiro". Esses itens dividem-se em três escalas gerais e 17 subescalas específicas. As escalas gerais são: problemas internalizantes (PI), problemas externalizantes (PE) e problemas totais (PT). Os resultados referentes às 17 subescalas específicas não foram analisados neste estudo.

A partir das pontuações obtidas nas respostas às questões do CBCL é possível analisar o grau de desvio da normalidade (faixa não clínica). A classificação do instrumento admite três faixas: a) clínica, indicadora de preocupações clínicas; b) limítrofe, indicadora de necessidade de acompanhamento para verificar possíveis demandas de intervenção; e c) não clínica, indicadora de bons recursos e pontos fortes do investigado (Bordin et al., 2013; Achenbach et al., 2016).

O diário de campo utilizado nesta pesquisa segue a proposta metodológica de Minayo (2014). Trata-se de um caderno de anotações no qual o pesquisador registra o que observa, assim como aquilo que não é objeto de nenhuma modalidade da entrevista e que, portanto, não pode ser acessado por meio de nenhum outro instrumento da pesquisa (Minayo, 2014). Nesta investigação, o diário de campo foi construído em forma de narrativas, com observações sobre os dias de levantamento de dados, características dos encontros com cada participante e o contexto das entrevistas (Oliveira, 2014).

\section{Procedimentos}

\section{Levantamento de dados}

O projeto de pesquisa foi aprovado pelo Comitê de Ética em Pesquisa da Universidade Federal de São Paulo (CEP 1283/2016). O levantamento de dados da pesquisa ocorreu entre novembro de 2016 e maio de 2017, na forma de entrevistas com o cuidador responsável pela criança ou adolescente com FC, em salas de espera de três CRTs da cidade de São Paulo (SP).

\section{Análise de dados}

Os dados do CBCL foram analisados via software Assessment data manager (ADM), versão 7.0, que é específico para análises dos instrumentos da bateria ASEBA. Esses dados foram sistematizados em dois grupos, segundo a variável faixa etária: grupo "criança” (6-11 anos) e grupo "adolescente" (12-18 anos).

Quanto aos escores, incluíram-se os do campo T score referentes a "Competências", "Problemas internalizantes (PI)" e "Problemas externalizantes (PE)". Os resultados foram classificados nas faixas "limítrofe/ clínica” (indicativas de necessidade de intervenção clínica) e "não clínica" (não indicativa de necessidade de intervenção clínica). As pontuações foram dispostas em planilha Excel para caracterização da amostra.

As narrativas do diário de campo foram tratadas via análise de conteúdo, segundo a proposta de Bardin (2011). As categorias de análise, elegidas a partir da leitura exaustiva do material, foram definidas a posteriori, considerando unidades de registro (temas).

\section{Resultados}

A constituição dos grupos de análise, "criança" e "adolescente", é mostrada na Tabela 1. Pacientes incluídos no grupo "criança" corresponderam a 71,0\% da amostra, enquanto os do grupo "adolescente", $29,0 \%$. Em relação ao sexo dos pacientes, o feminino foi o prevalente $(n=17)$.

\section{Tabela 1}

Distribuição da amostra, segundo idade e sexo do paciente.

\begin{tabular}{cccc}
\hline \multirow{2}{*}{ Idade } & \multicolumn{2}{c}{ Sexo } & \multirow{2}{*}{ Total } \\
\cline { 2 - 3 } & Masculino & Feminino & \\
\hline Criança & 9 & 13 & 22 \\
& $64,3 \%$ & $76,5 \%$ & $71,0 \%$ \\
Adolescente & 5 & 4 & 9 \\
& $35,7 \%$ & $23,5 \%$ & $29,0 \%$ \\
Total & 14 & 17 & 31 \\
& $100,0 \%$ & $100,0 \%$ & $100,0 \%$ \\
\hline
\end{tabular}




\section{Competências, problemas internalizantes e externalizantes, segundo CBCL}

A Tabela 2 mostra medidas descritivas (média, desvio padrão, máximo e mínimo) das pontuações obtidas nas escalas de competência e problemas psicológicos - problemas internalizantes (PI) e problemas externalizantes (PE), em cada grupo.

\section{Tabela 2}

Medidas descritivas das variáveis competência, problemas internalizantes (PI) e problemas externalizantes (PE), do CBCL, segundo os grupos de idade.

\begin{tabular}{ccccc}
\hline Grupo & & COMPETÊNCIA & PI & PE \\
\hline Criança & Média & $34,45^{*}$ & 59,45 & 58,50 \\
& Desvio- & 7,78 & 7,99 & 13,10 \\
& padrão & & & \\
& Mínimo & 23,00 & 39,00 & 33,00 \\
& Máximo & 50,00 & 73,00 & 77,00 \\
Adolescente & Média & $30,75^{*}$ & $63,56^{*}$ & 56,44 \\
& Desvio- & 5,20 & 13,13 & 11,65 \\
& padrão & & & \\
& Mínimo & 22,00 & 33,00 & 40,00 \\
& Máximo & 37,00 & 76,00 & 75,00 \\
\hline
\end{tabular}

Nota: Escores marcados com * pertencem à faixa clínica. $\mathrm{PI}=$ problemas internalizantes. $\mathrm{PE}=$ problemas externalizantes. $\mathrm{CBCL}=$ Child Behavior Checklist, $6-18$ (instrumento utilizado para levantamento desses dados).

No grupo "criança", somente a média relativa a competências foi em faixa clínica, enquanto no grupo "adolescente" as médias relativas a competências e a problemas internalizantes estiveram, ambas, em faixas clínicas.

\section{Análise do diário de campo}

A sistematização e análise dos dados do diário de campo indicou três categorias principais de conteúdo: a) Problemas de adesão ao tratamento; b) Demanda de atendimento psicológico; e c) O adoecimento enquanto experiência multideterminada.

\section{Problemas de adesão ao tratamento}

Nesta categoria, emergiram elementos referentes à falta de cooperação com o tratamento, do próprio paciente ou "do outro". Sobretudo na faixa etária da adolescência, os relatos de problemas na adesão ao tratamento referiram-se ao próprio paciente, seja via resistência às recomendações do tratamento ou simplesmente a não realização do recomendado pela equipe profissional, quando distantes da vigilância familiar. Em relação às crianças, tais problemas referiram-se essencialmente aos cuidadores - pessoas e instituições -, sendo os mais citados problemas relacionados ao "pai" e à "escola".

Alguns relatos da categoria "Problemas de adesão ao tratamento" indicaram reflexos de percepção dos adolescentes de sua condição de paciente crônico, evidente a si mesmo e aos outros. Os trechos dos relatos de anotações do diário de campo mostrados a seguir exemplificam essas características:

... [A mãe] conta que a filha não gosta de falar sobre a doença. Que poucos parentes sabem, amigos menos ainda. Que, por conta dessa questão, o tratamento é negligenciado, já que a menina não segue as recomendações quando na presença de pessoas que desconhecem sua doença. Que, quando amigos vãoà sua casa, é preciso esconder os remédios, que, inclusive, não os toma na presença de ninguém. A adolescência trouxe consigo novos desafios. Frases do tipo 'você acha que é tudo isso mesmo?', agora são parte dos questionamentos e discordâncias que a filha tem sobre seu tratamento (mãe de uma adolescente de 18 anos).

Outro fator apontado como prejudicial ao tratamento pelos cuidadores familiares foi a falta de cooperação institucional. Também compuseram essa categoria conteúdos sobre dificuldades encontradas nas interações com as escolas, no sentido de garantir o cumprimento do tratamento quando o paciente se encontrava em horário escolar.

Além dos problemas com os colegas, a mãe relata
problemas com os próprios profissionais da escola
que desconhecem a FC e não cooperaram com as
recomendações do tratamento quando o menino
está em horário escolar. Além disso, as internações
recorrentes são motivo de reclamações por parte
da escola (mãe de um adolescente de 13 anos).

\section{Demanda de atendimento psicológico}

$\mathrm{Na}$ categoria "Demanda de atendimento psicológico", foram incluídos os conteúdos referentes à necessidade desse tipo de atendimento profissional aos cuidadores/familiares ou aos próprios pacientes. Além disso, foi considerado se a demanda foi 
manifestada pelo próprio cuidador ou pela equipe profissional, via encaminhamento formal ou simples recomendação verbal. O conteúdo a seguir procura exemplificar essa condição:

Acabamos nos detendo mais às perguntas do CBCL, mas já próximo do fim da entrevista, ela [a mãe] fala que 'esse trabalho' [referindo-se à pesquisa] deveria ser mais com os pais das crianças do que propriamente com elas. 'Achoque eu que tinhaque passar emalgum lugar' (mãe de um menino de nove anos).

Conteúdo semelhante é expressado por outra participante, mãe de um paciente de sete anos, do sexo feminino, como se observa no seguinte trecho: "Começamos a entrevista e ela logo diz que tem que 'passar' mesmo no psicólogo, que as médicas já encaminharam a menina para esse atendimento". No entanto, neste caso, a participante também relatou que quem deveria "passar" com o psicólogo era a filha e que tal demanda fora sinalizada pela equipe profissional.

\section{$O$ adoecimento enquanto experiência multideterminada}

Na categoria "O adoecimento enquanto experiência multideterminada”, consideraram-se diversos conteúdos particulares aos contextos dos entrevistados que indicavam impacto no adoecimento. Incluiu-se nesta categoria questões referentes a dificuldades econômicas e sociais, enquanto limitadoras do acesso a bens e serviços essenciais à sobrevivência e ao tratamento; sobre a dinâmica familiar; sobre comorbidades e impactos de outras doenças, associadas ou não à FC; sobre o adoecimento e tratamento da criança/adolescente; e, ainda, relatos sobre limitações de realização de atividades e reflexos no corpo - aparência destoante de pares etários - provocados pela FC.

... por sugestão de uma médica, ela e o filho mudaram-se para SP em busca de um atendimento mais adequado. Além da FC, o menino apresenta uma série de alergias que limitam drasticamente sua alimentação. Ela conta que, no início, precisou morar na casa de amigos e, somente meses depois, o marido, juntamente ao filho caçula, puderam mudar-se para SP, também. Conta, inclusive, que todos já passaram por grave situação financeira, que só não passaram fome por conta do grupo da Igreja, próxima à casa da família, que fornecia alimentos a ele (mãe de um menino de dez anos).

Outra condição relatada por participantes referiu-se à falência do corpo que, mesmo submetido a um complexo tratamento, evoluía negativamente em suas funções, em decorrência da doença.

A mãe conta que em dado momento [da internação], a dor era tanta que ele chegou a falar que não tinha certeza se aquilo ia passar... Conta também que o filho sempre jogou futebol com os amigos $e$ que costumava cansar junto com eles, mas que de uns tempos para cá, estava cansando antes e isso o fez parar de jogar (mãe de um menino de 14 anos).

\section{Discussão}

A amostra deste estudo foi consonante com o levantamento da população brasileira de pessoas com FC, com maior incidência de pacientes na faixa etária infantil (GBEFC, 2017). Sobre a relação dos cuidadores familiares, o perfil dos participantes deste estudo corrobora os achados de outros trabalhos sobre o adoecimento em crianças e adolescentes com FC (Castro \& Piccinini, 2002; Pizzignacco, Mello, \& Lima, 2011; Afonso \& Mitre, 2013), no que diz respeito à maior participação da figura materna $(83,9 \%$ de frequência, neste estudo) como principal cuidadora na faixa inferior a 18 anos. O cuidado materno também é apontado, na literatura da área, como uma tarefa que requer recursos emocionais suficientes para suportar tanto suas próprias condições psicológicas quanto as possíveis dificuldades dos filhos em tratamento (Tavares et al., 2014).

Os resultados apresentados na Tabela 2 - as pontuações na faixa clínica das médias de competência em ambos os grupos e faixa clínica para problemas psicológicos do tipo internalizantes no grupo "adolescente" - são indicativos da prevalência de dificuldades emocionais (PI) em adolescentes com FC, em comparação com pacientes crianças. No estudo de Oliveira e Gomes (2004), meninas adolescentes apresentaram a menor adesão ao tratamento. Embora nesta pesquisa não tenham sido formados grupos a partir do sexo, o diálogo com os achados do estudo de Oliveira e Gomes (2004) dá indícios de uma possível relação entre problemas de adesão e questões emocionais (PI) em pacientes adolescentes. Tais problemas são 
possivelmente agravados pela deficiência de recursos de enfrentamento (competências), gradativamente mais exigidos desses pacientes com o passar do tempo.

Na infância, muitas das exigências do tratamento são ações de responsabilidade exclusiva dos cuidadores. No entanto, ao longo do desenvolvimento humano, espera-se do indivíduo maior autonomia diante de seus cuidados. No caso de pacientes de FC, essa maior autonomia associa-se a maiores responsabilidades com o próprio tratamento. Tal situação requer, do adolescente, recursos de enfrentamento, que às vezes lhe são insuficientes. Os resultados em faixas clínicas para competências, obtidos na análise do CBCL, devem ser interpretados nesse sentido, a partir da diferença entre um paciente de FC com poucos recursos de enfrentamento durante ainfância, eum adolescente com prejuízos em suas competências.

Além disso, os efeitos da doença/adoecimento da FC são mais evidentes e perceptíveis no corpo dos adolescentes. Ademais, seu maior desenvolvimento cognitivo, advindo da adolescência, leva a uma melhor compreensão de sua condição (Castro \& Piccinini, 2002).

Na categoria de análise intitulada "Problemas de adesão ao tratamento", os relatos sobre os adolescentes indicaram questionamentos em relação ao tratamento que pareciam dificultar a cooperação deles com as recomendações da equipe do CRT. Como exemplo, citou-se comportamentos de esconder a doença dos amigos e, em decorrência disso, deixar de tomar os medicamentos nos horários estipulados, ou mesmo não realizar exercícios respiratórios quando na presença de pessoas que desconheciam seu diagnóstico.

Para esses pacientes adolescentes, parecia ser difícil desenvolver a autonomia diante de seu tratamento também porque, tanto na equipe de saúde quanto na família, uma comunicação direta e clara com o próprio paciente não era priorizada. Esses achados corroboram, de certa forma, o apontado por Hess e Falcke (2013), que destacaram a importância de que as intervenções psicológicas considerem as relações familiares, no tratamento dos adolescentes.

Ainda na categoria "Problemas de adesão ao tratamento", relatos sobre dificuldades no diálogo com a escola apresentaram mais um fator de impacto no tratamento da FC. Essas dificuldades consistiam na recusa dos agentes escolares para a administração adequada de medicamentos ao paciente, na incompreensão quanto às faltas decorrentes dos períodos de internação e até em preconceitos de funcionários da instituição e dos colegas. Participantes também relataram dificuldades referentes à alimentação oferecida nas escolas aos pacientes de FC, haja vista que parte importante do tratamento dessa doença é decorrente da dieta especial do paciente. Porém, por ser doença rara, a FC é pouco conhecida socialmente e por vezes acaba negligenciada no importante espaço de desenvolvimento dessas crianças e adolescentes: a escola.

Os relatos da categoria "Demanda de atendimento psicológico" indicaram demandas pouco ou mal assistidas pelas instituições de saúde. Embora houvesse um pré-requisito de que as equipes de cuidado de FC, nos CRTs, fossem de natureza multiprofissional, essa condição não garantia a presença de um profissional psicólogo nas equipes onde ocorreu esta pesquisa. As demandas por atendimento psicológico não eram apenas referentes aos pacientes, mas também a seus cuidadores familiares, que, assim como eles, estavam inseridos no complexo contexto de adoecimento da FC.

Na categoria "O adoecimento enquanto experiência multideterminada", os relatos indicaram os custos do tratamento de FC como reorganizadores da dinâmica familiar - não só custos financeiros, como também emocionais. A necessidade constante de adaptação ao contexto da doença e às exigências do adoecimento, não só do paciente de FC, mas também de sua família, foi apontada em muitos dos relatos dos cuidadores.

\section{Considerações finais}

Dentro dos limites metodológicos deste estudo, que acena para a necessidade de aprofundamento em novas investigações, os resultados desta pesquisa mostraram uma preocupante situação quanto a indicativos de problemas psicológicos de crianças e adolescentes portadores de FC. A falta de avaliação psicológica específica, individual e familiar, e de consequentes e possíveis intervenções clínicas, incluindo aconselhamento psicológico, individual e familiar, foi uma lacuna identificada nas propostas multiprofissionais de atendimento à FC.

Prejuízos nos recursos de competências e/ou presença de problemas internalizantes (aqueles pouco perceptíveis pelos familiares e/ou pela equipe de saúde dos CRTs) caracterizam-se como fatores de ordem pouco propícia, ou até mesmo impeditiva, para o enfrentamento das dificuldades de pessoas com FC, em particular, na adolescência. Nesta investigação, os adolescentes se apresentaram mais prejudicados 
em termos de condições psicológicas pertinentes a competências e a problemas internalizantes, além de terem maior dificuldade na adesão ao tratamento.

A inserção escolar para pacientes com FC, conforme avaliada nesta pesquisa, mostrou uma séria deficiência quanto a políticas públicas de educação voltadas a essa população. Os problemas relativos às escolas avançaram para além da falta de dieta nutricional adequada, de ajuda na administração de medicamentos e de um cuidado para o desenvolvimento de condutas pró-sociais, nos colegas. $\mathrm{O}$ estudo indicou um despreparo geral dos agentes escolares para a educação, proteção e cuidado de crianças e adolescentes com FC, especialmente quanto ao manejo necessário face à ocorrência de faltas, nos períodos de internação.

Uma melhor qualificação no atendimento integral ao paciente com FC poderá advir, então, da oferta de atendimento psicológico no contexto das equipes de saúde especializadas, além de intervenções permanentes com as escolas que também atuam com crianças e adolescentes acometidos por essa doença. Assim, poderão ser aumentadas as oportunidades de assegurar condições mínimas e necessárias aos pacientes de FC, quanto ao desenvolvimento positivo em sua vida pessoal, familiar e social.

\section{Referências}

Achenbach, T. M. (2015). Multicultural evidence-based assessment using the Achenbach System of Empirically Based Assessment (ASEBA) for ages 1/2-90+. Psychologia: Avances de la Disciplina, 9(2), 13-23.

Achenbach, T. M., Ivanova, M. Y., Rescorla, L. A., Turner, L. V., \& Althoff, R. R. (2016). Internalizing/Externalizing problems: Review and recommendations for clinical and research applications. Journal of the American Academy of Child \& Adolescent Psychiatry, 55(8), 647-656. https:// doi.org/10.1016/j.jaac.2016.05.012

Achenbach, T. M., \& Rescorla, L. A. (2001). Manual for the ASEBA school, age forms \& profiles. University of Vermont; Research Center for Children, Youth \& Families.

Achenbach, T. M., \& Rescorla, L. A. (2010). Mental health practitioners' guide for the Achenbach System of Empirically Based Assessment (ASEBA) (7a ed.) [Manual]. University of Vermont; Research Center for Children, Youth, \& Families.

Adde, F. V. (2015). Nutrição em fibrose cística: Tão importante quanto o manejo da doença pulmonar. Revista Paulista de Pediatria, 33(1), 1-2. https://doi.org/10.1016/j.rpped.2014.12.001

Afonso, S. B. C., Gomes, R., \& Mitre, R. M. A. (2015). Narrativas da experiência de pais de crianças com fibrose cística. Interface - Comunidade, Saúde, Educação, 19(55), 1077-1088. http://doi.org/10.1590/1807-57622014.0569

Afonso, S. B. C., \& Mitre, R. M. A. (2013). Notícias difíceis: Sentidos atribuídos por familiares de crianças com fibrose cística. Ciência e Saúde Coletiva, 18(9), 2605-2613. http://doi.org/10.1590/S1413-81232013000900015

Athanazio, R. A., Silva Filho, L.V. R., Vergara, A. A., Ribeiro, A. F., Riedi, C. A., Procianoy, E. F. A., Adde, F.V., Reis, F J. C., Ribeiro, J. D., Torres, L. A., Fuccio, M. B., Epifanio, M., Firmida, M. C., Damaceno, N., Ludwig-Neto, N., Maróstica, P. J. C., Rached, S. Z., \& Melo, S. F. O. (2017). Diretrizes brasileiras de diagnóstico e tratamento da fibrose cística. Jornal Brasileiro de Pneumologia, 43(3), 219-245. http:// doi.org/10.1590/S1806-37562017000000065

Bardin, L. (2011). Análise de conteúdo (L. Antero Reto, A. Pinheiro, Trads.). Edições 70.

Bordin, I. A., Rocha, M. M., Paula, C. S., Teixeira, M. C. T. V., Achenbach, T. M., Rescorla, L. A., \& Silvares, E. F. M. (2013). Child Behavior Checklist (CBCL), Youth Self Report (YSR) e Teacher's Report Form (TRF): Uma visão geral sobre o desenvolvimento das versões originais e brasileiras. Cadernos de Saúde Pública, 29(1), 13-28.

Borges, C. C., Bezerra, A. C., \& Scaff, D. R. A. (2019). Histórico de implantação de um centro de referência no tratamento da fibrose cística e uma experiência de interconsultas com a Psicologia. Visão Acadêmica, 20(3), 124-135.

Cabello, G. M. K. (2011). Avanços da genética na fibrose cística. Revista do Hospital Universitário Pedro Ernesto, 10(40), 36-45.

Castro, E. K., \& Piccinini, C. A. (2002). Implicações da doença orgânica crônica na infância para as relações familiares: Algumas questões teóricas. Psicologia: Reflexão e Crítica, 15(3), 625-635. http://doi.org/10.1590/ S0102-79722002000300016

Chaves, C. R. M. M., Cunha, A. L. P., Costa, A. C., Costa, R. S. S., \& Lacerda, S. V. (2015). Estado nutricional e distribuição de gordura corporal em crianças e adolescentes com fibrose cística. Ciência e Saúde Coletiva, 20(11), 3319-3328. http://doi.org/10.1590/1413-812320152011.03242015 
Conto, C. L., Vieira, C. T., Fernandes, K. N., Jorge, L. M., Cândido, G. S., Barbosa, R. I., \& Dutra, R. C. (2014). Prática fisioterapêutica no tratamento da fibrose cística. ABCS Health Sciences, 39(2), 96-100. http://doi.org/10.7322/ abcshs.v39i2.629

Cystic Fibrosis Foundation. (2018). 2018 Patient Registry Annual Data Report.

D’Alessandro, V., Rentería, F., Fernández, A., Martínez, M. I., \& Segal, E. (2009). Comparación del estado clínico-funcional en niños con fibrosis quística detectados por pesquisa neonatal o por sintomas clínicos. Archivos Argentinos de Pediatria, 107(5), 430-435.

Griese, M., \& Kappler, M. (2008). Melhora efetiva no tratamento da fibrose cística através da aplicação de medidas elementares: Lições essenciais não apenas para países em desenvolvimento. Jornal de Pediatria, 84(5), 383-385. http://doi.org/10.1590/S0021-75572008000600002

Grupo Brasileiro de Estudos de Fibrose Cística. (2015). Registro brasileiro de fibrose cística 2015.

Grupo Brasileiro de Estudos de Fibrose Cística. (2016). Registro brasileiro de fibrose cística 2016.

Grupo Brasileiro de Estudos de Fibrose Cística. (2017). Registro brasileiro de fibrose cística 2017.

Hess, A. R. B., \& Falcke, D. (2013). Sintomas internalizantes na adolescência e as relações familiares: Uma revisão sistemática da literatura. Revista Psico-USF, 18(2), 263-276. http://doi.org/10.1590/S1413-82712013000200010

Lentini, E., López-Millán, A., Lores, A. M., Pesciullesi, M. R., Ituarte, L. M., \& Vieira, T. B. (2014). Fibrosis quística: Aumento de la sobrevida en un centro especializado a 10 años de seguimiento. Revista Chilena de Pediatria, 85(3), 281-287. http://doi.org/10.4067/S0370-41062014000300003

Mello, D. B., \& Moreira, M. C. N. (2010). A hospitalização e o adoecimento pela perspectiva de criança e jovens portadores de fibrose cística e osteogênese imperfeita. Ciência e Saúde Coletiva, 15(2), 453-461. https://doi.org/ $10.1590 /$ S1413-81232010000200022

Minayo, M. C. S. (2014). O desafio do conhecimento: Pesquisa qualitativa em saúde (14a ed.). Hucitec.

Oliveira, R. C. M. (2014). (Entre)linhas de uma pesquisa: O diário de campo como dispositivo de (in)formação na/da abordagem (Auto)biográfica. Revista Brasileira de Educação de Jovens e Adultos, 2(4), 69-87.

Oliveira, V. Z., \& Gomes, W. B. (2004). Comunicação médico-paciente e adesão ao tratamento em adolescentes portadores de doenças orgânicas crônicas. Estudos de Psicologia, 9(3), 459-469. http://doi.org/10.1590/ S1413-294X2004000300008

Pizzignacco, T. P., Mello, D. F., \& Lima, R. G. (2011) A experiência da doença na fibrose cística: Caminhos para o cuidado integral. Revista da Escola de Enfermagem da USP, 45(3), 638-644. https://doi.org/10.1590/ S0080-62342011000300013

Quittner, A. L., Abbott, J. Georgiopoulos, A. M. Goldbeck, L., Smith, B., Hempstead, S. E., Marshall, B., Sabadosa, K. A., Elborn, S., \& The International Committee on Mental Health. (2016). International committee on mental health in cystic fibrosis: Cystic fibrosis foundation and European cystic fibrosis society consensus statements for screening and treating depression and anxiety. Thorax, 71(1), 1-9. http:// doi.org/10.1136/thoraxjnl-2015-207488

Tavares, K. O., Carvalho, M. D. B., \& Pelloso, S. M. (2010). O que é ser mãe de uma criança com fibrose cística. Revista Gaúcha de Enfermagem, 31(4), 723-729. http://doi.org/10.1590/S1983-14472010000400016

Tavares, K. O., Carvalho, M. D. B., \& Pelloso, S. M. (2014). Dificuldades vivenciadas por mães de pessoas com fibrose cística. Texto \& Contexto: Enfermagem, 23(2), 294-300. http:// doi.org/ 10.1590/0104-07072014000050013

\section{Carla Cristina Borges}

Psicóloga, mestra em Ciências pela Universidade Federal de São Paulo (Unifesp), Santos - SP, Brasil. Pesquisadora do Laboratório de Psicologia Ambiental e Desenvolvimento Humano da Unifesp (LADH/Unifesp), Santos - SP, Brasil.

E-mail: carlaborges.psi@gmail.com

(D) https://orcid.org/0000-0002-6912-1826 


\section{Nancy Ramacciotti de Oliveira-Monteiro}

Psicóloga pela Faculdade de Filosofia, Ciências e Letras da Universidade Católica de Santos (Unisantos), Santos SP, Brasil. Mestra em Psicologia Social pela Pontifícia Universidade Católica de São Paulo (PUCSP), São Paulo - SP, Brasil. Cursou doutorado e pós-doutorado em Psicologia Social pela Universidade de São Paulo (USP), São Paulo-SP, Brasil. Professora Associada da Universidade Federal de São Paulo, campus Baixada Santista (Unifesp-BS), Santos - SP, Brasil.

E-mail: nancy.unifesp@gmail.com

(D) https://orcid.org/0000-0002-8963-5162

Endereço para envio de correspondência:

Universidade Federal de São Paulo, campus Baixada Santista, Edifício Central. Rua Silva Jardim, 136, sala 327, Bairro Vila Mathias. CEP: 11015-020. Santos - SP. Brasil.

Recebido $30 / 01 / 2020$

Aceito 04/05/2021

Received 01/30/2020

Approved 05/04/2021

Recibido 30/01/2020

Aceptado 04/05/2021

Como citar: Borges, C. C., \& Oliveira-Monteiro, N. R. de. (2022). Problemas internalizantes e externalizantes de crianças e adolescentes com fibrose cística. Psicologia: Ciência e Profissão, 42, 1-11.

https://doi.org/10.1590/1982-3703003233513

How to cite: Borges, C. C., \& Oliveira-Monteiro, N. R. de. (2022). Internalizing and externalizing problems of children and adolescents with cystic fibrosis. Psicologia: Ciência e Profissão, 42, 1-11.

https://doi.org/10.1590/1982-3703003233513

Cómo citar: Borges, C. C., \& Oliveira-Monteiro, N. R. de. (2022). Problemas de internalización y externalización de niños y adolescentes con fibrosis quística. Psicologia: Ciência e Profissão, 42, 1-11.

https://doi.org/10.1590/1982-3703003233513 